\title{
Visual functioning and quality of life outcomes among cataract operated and unoperated blind populations in Nepal
}

\author{
G P Pokharel, S Selvaraj, Leon B Ellwein
}

\begin{abstract}
Background-Visual acuity and vision related quality of life outcomes in cataract surgery were evaluated in a population based survey in two geographic zones in Nepal.

Methods-Case finding was based on random sampling using a stratified cluster design with door to door enumeration of people aged $\geqslant 45$ years followed by eye examinations at village sites. All aphakics/ pseudophakics, those with visual acuity less than $6 / 60$ in either eye, and a sample of those with normal visual acuity were administered visual functioning (VF) and quality of life (QOL) questionnaires. Results-15\% of the 159 cataract operated cases had presenting visual acuity $\geqslant 6 / 18$ in both eyes, $38 \%$ with best corrected visual acuity. $21 \%$ were still blind with presenting visual acuity $<6 / 60$ in both eyes, $7 \%$ with best correction. On a 0-100 scale, mean VF and QOL scores were 87.2 and 93.9 respectively in normally sighted unoperated individuals, dropping to 15.6 and 29.5 for those severely blind $(<3 / 60)$. Among the cataract operated, mean VF and QOL scores were 47.5 and 55.4 , respectively. VF and QOL scores correlated with vision status at statistically significant levels (p $<0.0001$ )
\end{abstract}

Conclusion-Cataract surgery outcomes, whether measured by traditional visual acuity or by patient reported VF/QOL, are at levels many would consider unacceptably low. It is apparent that in the quest to reduce cataract blindness much more attention must be given to improving surgery outcomes.

(Br F Ophthalmol 1998;82:606-610)

Himalaya,

Kathmandu, Nepal

G P Pokharel

Aravind Eye Hospital, Madurai, India

S Selvaraj

National Eye Institute, Bethesda, Maryland, USA

Leon B Ellwein

Correspondence to: Leon B Ellwein, PhD, National Eye Institute, 31 Center Drive, MSC 2510, Bldg 31, Rm 6A-04, Bethesda, MD 20892-2510, USA.

Accepted for publication 9 January 1998 ision restored. The main points of current debate deal indirectly with this assumption in raising questions of appropriate technology, the extent to whether ECCE/IOL surgery should be favoured over ICCE with spectacles, and whether backlog cases should be given priority over newly blind cases, including those who have not yet progressed beyond unilateral blindness. ${ }^{4}$

We advocate that attention should be redirected towards ensuring that successful outcomes are indeed being realised to the greatest extent possible. This entails, as a minimum, patient follow up to measure the extent to which surgery has restored visual acuity, visual functioning and related quality of life. Developing the understanding and wherewithal necessary to minimise unsatisfactory outcomes (and thus minimising the wasting of eye care resources, not to mention patient welfare) is particularly critical in developing country settings, where extreme limitations on resource availability frequently limit the number of cases that can be offered cataract surgery. We recognise that greater attention will not be given to cataract surgery outcomes until convincing evidence shows that improvement is needed. The results reported here help to make this point.

\section{Methods}

The study was conducted in the Lumbini and Bheri zones of western Nepal. Both zones have active prevention of blindness programmes that include outreach activities, such as cataract screening and surgery camps. The Lumbini eye care programme is based at the Rana-Ambika Eye Hospital in Bhairahawa (Rupandehi district) with eye clinics in four of the other five zone districts. It was established in 1985 with support from the SEVA Foundation. The Bheri eye care programme was started in 1983 by the Swiss Red Cross. It includes the Fateh-Bal Eye Hospital in Nepalgunj (Banke district) with eye clinics in each of the four other zone districts. Both institutions have established a reputation as models of cost effective eye care services, suggesting that cataract surgery outcomes in the Lumbini and Bheri zones should be typical of, or even better than, what one would generally find in developing country settings.

The study population consisted of a randomly drawn sample of 4602 individuals, aged 45 years or older, distributed across all districts (six in Lumbini and five in Bheri zone). Within each district, cluster sampling of village and municipality wards with an estimated 75-150 study subjects each was employed. The field- 
Table 1 Vision status of cataract operated individuals based on presenting and best corrected visual acuity

\begin{tabular}{|c|c|c|c|c|}
\hline \multirow[b]{2}{*}{ Vision category } & \multicolumn{2}{|c|}{ Presenting visual acuity } & \multicolumn{2}{|c|}{ Best corrected visual acuity } \\
\hline & Aphakics & Pseudophakics & Aphakics & Pseudophakics \\
\hline Normal/near normal ( $\geqslant 6 / 18$ both eyes) & $21(15.8)$ & $3(11.5)$ & $53(39.8)$ & $7(26.9)$ \\
\hline Visual impairment ( $\geqslant 6 / 60$ better eye, $<6 / 18$ to $\geqslant 6 / 60$ worse eye) & $21(15.8)$ & $8(30.8)$ & $14(10.5)$ & $5(19.2)$ \\
\hline Unilateral blindness ( $\geqslant 6 / 60$ better eye, $<6 / 60$ worse eye) & $58(43.6)$ & $12(46.2)$ & $55(41.4)$ & $12(46.2)$ \\
\hline Moderate blindness ( $<6 / 60$ to $\geqslant 3 / 60$ better eye, $<6 / 60$ worse eye) & $10(6.3)$ & 0 & $1(0.8)$ & 0 \\
\hline Severe blindness ( $<3 / 60$ both eyes) & $22(16.5)$ & $2(7.7)$ & $9(6.8)$ & $1(3.8)$ \\
\hline Missing information & $1(0.8)$ & $1(3.8)$ & $1(0.8)$ & $1(3.8)$ \\
\hline All & $133(100.0)$ & $26(100.0)$ & $133(100.0)$ & $26(100.0)$ \\
\hline
\end{tabular}

work in each zone was carried out by a separate team. Enumerators mapped and numbered all ward households, followed by door to door visits to identify by name all residents $\geqslant 45$ years of age and to invite them to a village examination site. At the examination site, visual acuity measurements, vision functioning (VF) and quality of life (QOL) interviews, and eye examinations were conducted. Methods used in drawing the random sample and in examining patients are described in the companion paper. ${ }^{1}$

VF/QOL INTERVIEWING

All people with presenting visual acuity of $<6 / 60$ in either eye were referred for VF/QOL interviews. All cataract surgery suspects were also interviewed, regardless of visual acuity levels. Additionally, a one in 20 sample of individuals with normal or near normal vision $(\geqslant 6 / 18$ in both eyes) was administered the $\mathrm{VF} / \mathrm{QOL}$ questionnaires. (Wording in the QOL questionnaire is not appropriate for individuals not experiencing a vision problem, and so it was not administered to the full normal sample.) Interviews were administered by one of two team interviewers in a private area within the examination site. The interview episode ranged from 10 to 20 minutes in duration. All but five of the people who agreed to be interviewed were able to complete the entire interview process.

The VF/QOL questionnaires used in this study originated in a large scale clinical trial of the comparative safety and efficacy of ICCE and ECCE in Madurai, India. ${ }^{5}$ The 13 item VF questionnaire includes one general question assessing overall vision and four subscales: a visual perception subscale (activity limitation, near vision, intermediate vision, distance vision), a single question peripheral vision subscale, a sensory adaptation subscale (light/dark adaptation, visual search, colour discrimination, glare disability), and a single question depth perception subscale. The 12 item QOL questionnaire contains four subscales: self care (bathing, eating, dressing, toiletting), mobility (walking to neighbours, walking to shops,

Table 2 Visual acuity in operated eyes

\begin{tabular}{|c|c|c|c|c|c|c|}
\hline \multirow[b]{2}{*}{ Presenting } & \multicolumn{5}{|c|}{ Best corrected } & \multirow[b]{2}{*}{ Total } \\
\hline & $\geqslant 6 / 18$ & $<6 / 18-\geqslant 6 / 60$ & $<6 / 60-\geqslant 3 / 60$ & $<3 / 60$ & Missing & \\
\hline$\geqslant 6 / 18$ & 92 & & & & & $92(41.8)$ \\
\hline$<6 / 18-\geqslant 6 / 60$ & 36 & 21 & & & & $57(25.9)$ \\
\hline$<6 / 60-\geqslant 3 / 60$ & 17 & 1 & 2 & & 1 & $21(9.5)$ \\
\hline$<3 / 60$ & 13 & 12 & 1 & 20 & & $46(20.9)$ \\
\hline Missing & & & & 1 & 3 & $4(1.8)$ \\
\hline Total & $158(71.8)$ & $34(15.5)$ & $3(1.4)$ & $21(9.5)$ & $4(1.8)$ & $220(100)$ \\
\hline
\end{tabular}

doing household chores), social interaction (attending functions, meeting friends), and mental wellbeing (burden on others, dejection, loss of confidence).

English versions of these two questionnaires were translated into Nepali and four Nepalese dialects. The questionnaires were back translated to English by an independent translator. Minor modifications of wording in the translated versions were then made as necessary to improve the concordance between English and Nepalese versions. Before use in interviewer training, the questionnaires were administered to several hospital outpatients to ensure comprehension of each question.

VF/QOL QUALITY ASSURANCE

Interviewers were trained before the start of the study at the Nepal Eye Hospital in Kathmandu over a 2 week period. Trainees practised on hospital eye care patients after being familiarised with interviewing techniques through role playing. A pilot study was carried out in three areas in Bheri zone, which were not part of the selected study areas.

Intraobserver and interobserver variation studies were conducted for each VF/QOL question during the pilot for the four interviewers. Patients were re-interviewed after a 1 hour wait. The intraobserver studies involved 20 patients (most with visual acuity $<6 / 60$ in at least one eye) for each interviewer. Interobserver studies were done in a similar number of patients for each team. For one of the interviewers, intraobserver kappa statistics were below 0.4 (fair to poor agreement) for three of the $13 \mathrm{VF}$ questions. The next worst interviewer had no kappas below 0.4, with 12 between 0.4 and 0.75 . For the best interviewer, 11 of the 13 questions had very good agreement with kappas above 0.75 . For the 12 item QOL questionnaire, the worse performing interviewer had four questions with kappas below 0.4 , the next worst had 2 below 0.4 . The two worst performing interviewers were from the same team (Lumbini). Interobserver agreement for this team pair was below 0.4 for 12 of the VF questions and 10 of the QOL questions (17 patients). The other team pair had one question below 0.4 on the $\mathrm{VF}$ questionnaire and 8 on the QOL questionnaire (18 patients). Even after further training, one interviewer did not show sufficient improvement during a mid survey monitoring and was removed from the Lumbini team (without replacement).

DATA MANAGEMENT AND ANALYSIS

Completed VF/QOL forms were sent to Kathmandu for double data entry by independent 
Table 3 Principal cause of impaired vision/blindness in operated eyes

\begin{tabular}{lrcr}
\hline & \multicolumn{3}{c}{ Best corrected visual acuity } \\
\cline { 2 - 4 } Cause & $<6 / 18-\geqslant 6 / 60$ & $<6 / 60$ & Total \\
\hline Surgical complications & 14 & 8 & 22 \\
Macular degeneration & 9 & 4 & 9 \\
Optic atrophy & & 4 & 4 \\
Glaucoma & 3 & 3 & 6 \\
Posterior capsule & & & \\
$\quad$ opacification & 2 & 1 & 3 \\
Corneal scar & 6 & 1 & 1 \\
Amblyopia & 34 & 24 & 98 \\
Undetermined/other & & & \\
All & & & \\
\hline
\end{tabular}

clerks. A matching program was used in the identification and resolution of data entry discrepancies. Total VF and QOL scores were calculated as an equally weighted average of the four subscale scores. (The VF score does not include the general vision question.) Within subscales, questions are also weighted equally. The association between VF/QOL scores and visual acuity was examined using the non-parametric Kruskal-Wallis test. Analysis of variance was used to evaluate whether VF and QOL scores differed among cataract operated and unoperated groups. Effect sizes were computed for group comparisons to help interpret the practical importance of score differences. Univariate and multivariate regression analyses were undertaken to quantify the association of sociodemographic characteristics with $\mathrm{VF} / \mathrm{QOL}$ scores.

\section{Results}

CLINICAL OUTCOMES

In all, 159 cataract operated individuals (133 aphakics and 26 pseudophakics) were identified in the survey. Table 1 presents vision status based on both presenting and best corrected visual acuity for these individuals; 41 of 133 aphakics and 14 of 26 pseudophakics had presenting visual acuity $\geqslant 6 / 18$ in the operated eye (worse eye if bilaterally operated).

Presenting visual acuity is with the usual correction if the patient presents with glasses. Of the 22 aphakics in the severe blindness category, only three out of the 18 with spectacle information reported were wearing glasses. For the other vision categories, 69 of 85 were wearing glasses. The best corrected visual acuity

Table 4 Visual functioning and quality of life mean scores (SD) by presenting vision status in unoperated individuals

\begin{tabular}{|c|c|c|c|c|}
\hline \multirow[b]{2}{*}{$V F$ scales } & \multicolumn{4}{|l|}{ Vision status } \\
\hline & $\begin{array}{l}\text { Normal } \\
(n=140)\end{array}$ & $\begin{array}{l}\text { Unilateral } \\
\text { blindness } \\
(n=204)\end{array}$ & $\begin{array}{l}\text { Moderate } \\
\text { blindness }(n=92)\end{array}$ & $\begin{array}{l}\text { Severe blindness } \\
(n=93)\end{array}$ \\
\hline Vision perception & $88.3(14.3)$ & $62.0(28.0)$ & $28.1(26.5)$ & $14.4(22.0)$ \\
\hline Peripheral vision & $93.6(15.9)$ & $60.8(30.1)$ & $25.7(32.1)$ & $12.2(24.0)$ \\
\hline Sensory adaptation & $81.9(13.3)$ & $60.5(25.6)$ & $28.6(29.2)$ & $16.1(24.3)$ \\
\hline Depth perception & $97.9(10.7)$ & $80.2(30.8)$ & $38.1(39.0)$ & $21.9(33.5)$ \\
\hline Total VF & $87.2(11.3)$ & $63.2(25.0)$ & $28.9(28.0)$ & $15.6(23.0)$ \\
\hline QOL scales & $(n=102)$ & $(n=204)$ & $(n=92)$ & $(n=93)$ \\
\hline Self care & $97.5(7.7)$ & $84.5(26.6)$ & $47.9(40.9)$ & $33.6(37.8)$ \\
\hline Mobility & $92.2(13.5)$ & $69.0(33.1)$ & $35.5(38.4)$ & $22.6(30.1)$ \\
\hline Social & $93.5(12.9)$ & $73.2(30.8)$ & $52.2(38.0)$ & $33.0(38.1)$ \\
\hline Mental & $91.0(15.3)$ & $66.9(32.1)$ & $42.4(35.3)$ & $28.6(34.0)$ \\
\hline Total QOL & $93.9(9.5)$ & $74.3(25.8)$ & $44.1(33.6)$ & $29.5(31.1)$ \\
\hline
\end{tabular}

results show that a significant number of cases would have improved vision if refractive errors were fully corrected.

The improvement in vision with refraction is explored in further detail by separately considering each of the 220 operated eyes (Table 2). Thirty one eyes were pseudophakic, 16 with presenting visual acuity $\geqslant 6 / 18$. Eighty of the 124 eyes $(64.5 \%)$ with presenting visual acuity $<6 / 18$ improve by at least one visual acuity category with best correction. One hundred fifty eight, or almost three quarters, of the operated eyes reach the normal/near normal level $(\geqslant$ $6 / 18)$.

Of the 58 operated eyes with best corrected visual acuity $<6 / 18$ (Table 2), surgical complications, and macular degeneration are the most common identified causes (Table 3). Because we are dealing with best corrected visual acuity, refractive error as a principal cause does not arise. Except for laser capsulotomy for those eyes with capsule opacification, it is not likely that vision can be restored beyond that possible with refraction.

VISUAL FUNCTION/QUALITY OF LIFE OUTCOMES Visual functioning and quality of life interviews were conducted in 153 of the 159 cataract operated individuals (six individuals were missed), in 204 of 270 unoperated individuals with unilateral blindness, in 92 of 95 unoperated individuals with moderate blindness, and in 93 of 112 unoperated individuals with severe blindness. In addition, 140 individuals with normal or near normal vision were interviewed for VF and 102 for QOL (the normal controls).

Responses in both VF and QOL questionnaires had high internal consistency. For the total VF scale, the Cronbach alpha was 0.97 (p $<0.0001$ ), with item total correlations ranging from 0.66 to 0.88 . For the total QOL scale, the Cronbach alpha was also $0.97(p<0.0001)$, with item total correlation ranging from 0.78 to 0.90 .

Table 4 presents total and subscale VF and QOL mean scores for the unoperated individuals according to presenting vision status. The association between mean scores and vision status is very strong for each VF and QOL subscale (Kruskal-Wallis p <0.0001).

In linear regression analysis, $60.8 \%$ of the observed variability in total VF scores can be explained by vision status alone. Adding sociodemographic variables in a multiple regression model explains $66.5 \%$ of score variance. In addition to vision status, age, and zone are statistically significant (Wald test, $\mathrm{p}<0.0001$ ); sex $(\mathrm{p}=0.130)$, literacy $(\mathrm{p}=0.793)$, and urban/rural area $(\mathrm{p}=0.706)$ are not. Regression analysis of total QOL scores yields similar results: vision status alone explains $42.3 \%$ of the observed score variability. With multiple regression, $50.5 \%$ of the variability is explained, along with vision status, by age ( $\mathrm{p}$ $<0.0001)$, zone $(\mathrm{p}<0.0001)$, and $\operatorname{sex}(\mathrm{p}=$ $0.011)$ at statistically significant levels; literacy $(\mathrm{p}=0.408)$ and area $(\mathrm{p}=0.356)$ are not significant. VF and QOL scores decrease with older age, residence in Bheri zone, and female 
Table 5 Visual functioning and quality of life mean scores (SD) by presenting vision status in cataract operated individuals

\begin{tabular}{lllllll}
\hline & \multicolumn{1}{l}{ Vision status } & & & & \\
\cline { 2 - 6 } VF scales & Normal $(n=23)$ & $\begin{array}{l}\text { Vision impairment } \\
(n=28)\end{array}$ & $\begin{array}{l}\text { Unilateral } \\
\text { blindness }(n=69)\end{array}$ & $\begin{array}{l}\text { Moderate blindness } \\
(n=10)\end{array}$ & $\begin{array}{l}\text { Severe blindness } \\
(n=23)\end{array}$ & All (n=153) \\
\hline $\begin{array}{llllll}\text { Vision perception } \\
\text { Peripheral vision }\end{array}$ & $75.4(25.8)$ & $60.1(25.9)$ & $53.0(29.6)$ & $28.3(28.9)$ & $8.0(18.9)$ & $49.3(33.6)$ \\
Sensory adaptation & $65.6(23.7)$ & $56.0(37.5)$ & $49.8(34.6)$ & $23.3(31.6)$ & $5.8(16.4)$ & $46.2(38.6)$ \\
Depth perception & $84.1(28.2)$ & $78.6(37.1)$ & $44.9(26.8)$ & $28.3(30.2)$ & $5.8(18.4)$ & $43.2(31.2)$ \\
Total VF & $72.2(23.3)$ & $60.1(25.6)$ & $62.8(37.3)$ & $36.7(42.9)$ & $10.1(25.5)$ & $59.3(41.9)$ \\
\hline QOL scales & $(n=23)$ & $(n=28)$ & $(n=69)$ & $(n=10)$ & $(n=23)$ & $(n=153)$ \\
\hline Self care & $81.2(32.3)$ & $74.4(32.9)$ & $68.0(36.2)$ & $45.0(43.6)$ & $14.1(27.3)$ & $61.6(40.2)$ \\
Mobility & $83.6(29.2)$ & $64.7(33.7)$ & $55.7(38.3)$ & $31.1(41.2)$ & $9.7(23.3)$ & $53.0(40.6)$ \\
Social & $62.3(41.2)$ & $63.7(37.7)$ & $58.5(37.2)$ & $45.0(36.9)$ & $26.8(36.5)$ & $54.4(39.3)$ \\
Mental & $74.4(34.7)$ & $60.7(39.1)$ & $51.4(35.9)$ & $31.1(33.9)$ & $18.4(32.8)$ & $50.3(39.1)$ \\
Total QOL & $76.9(28.8)$ & $66.8(30.5)$ & $59.2(33.9)$ & $38.1(35.8)$ & $16.2(24.3)$ & $55.4(36.2)$ \\
\hline
\end{tabular}

sex. It is not clear whether the significance of zone is actually the result of geographic differences or the comparative influence of the interview team.

Total and subscale VF and QOL mean scores for cataract operated cases are presented in Table 5. Here, too, the association between presenting vision status and mean scores for each VF and QOL subscale is strong (K-W, p $<0.0001$ except for the social subscale $\mathrm{p}=$ $0.0058)$. Vision status explains $38.7 \%$ of the observed variability in total $\mathrm{VF}$ scores and $26.9 \%$ of variability in total QOL scores. Considering the influence of sociodemographic variables alongside visual status explains $55.3 \%$ of the variance for VF scores and $39.7 \%$ for QOL scores. In addition to visual status, age $(\mathrm{p}<0.001)$ and zone $(\mathrm{p}<0.001)$ are statistically significant for $V F$ with age $(p=0.002)$ and zone $(p<0.001)$ also significant for QOL. As with the unoperated, VF and QOL scores decrease with older age and residence in Bheri zone.

Comparison of total and subscale VF and QOL mean scores for unoperated versus operated groups within each vision category reveals that the operated mean scores are consistently lower. As a group, the cataract operated individuals score between the unoperated with unilateral blindness and the unoperated with moderate blindness. Meaningful statistical testing for differences between operated and unoperated groups within vision categories is limited by the large number of possible comparisons (one or more differences may be statistically significant due to chance alone) and by possible confounding in any nonrandomised comparison due to socioeconomic factors (beyond differences in age, sex, and literacy) of an unknown nature and magnitude.

The interpretation of differences between VF/QOL scores is addressed in Table 6 in

Table 6 Interpretation of differences in visual functioning and quality of life scores among unoperated and operated groups

\begin{tabular}{llllll}
\hline & \multicolumn{2}{c}{ Target state $(V F$ effect size) } & & \multicolumn{2}{c}{ Target state (QOL effect size) } \\
\cline { 2 - 3 } $\begin{array}{lllll}\text { Cataract } \\
\text { Baseline state }\end{array}$ & Normal vision & & $\begin{array}{l}\text { Cataract } \\
\text { operated }\end{array}$ & Normal vision \\
\hline Severely impaired & -0.6 & 1.0 & & -0.7 & 0.8 \\
Cataract operated & 0.0 & 1.2 & & 0.0 & 1.1 \\
Moderate blindness & 0.7 & 2.1 & & 0.3 & 1.5 \\
Severe blindness & 1.4 & 3.1 & & 0.8 & 2.1 \\
\hline
\end{tabular}

terms of effect size. ${ }^{6}$ Effect size is calculated as the difference between the target and baseline vision state mean scores divided by the baseline standard deviation. Effect sizes of 0.3 or less are considered small, those around 0.5 moderate, and those above 0.8 large. Accordingly, the analysis suggests that a moderate improvement in quality of life and visual functioning is achieved by operating on cataract cases with moderate blindness and a large improvement in cases with severe blindness. If normal vision were to be achieved through cataract surgery, both VF and QOL effect sizes would be very large. It should be recognised that the cross sectional comparisons in Table 6 are between groups with somewhat different sociodemographic composition, and not representative of actual longitudinal change within patients; thus, they are at best only suggestive of what might be achieved by moving from one vision state to another.

\section{Discussion}

It is likely that both clinical and VF/QOL outcomes in cataract operated patients are below what many would expect. Visual acuity and VF/QOL deficits cannot be attributed primarily to cataract impairment in an unoperated fellow eye. A significant part of the problem lies with incomplete vision restoration in already operated eyes. One can only speculate on what would have been achieved if greater priority had been given at the outset to maximising outcomes.

One important cause of impairment in the cataract operated patient amenable to solution is refractive error. The substantial improvement in vision among operated individuals possible with refractive error correction points to the need for such follow up services among both aphakics and pseudophakics. Many of these people have less than satisfactory, but correctable vision. Unfortunately, they may be attributing their loss of vision to a failure of cataract surgery, even though this may not be the case, and discouraging others from seeking such treatment.

The other causes of impairment in cataract operated eyes are less tractable. The operated cases represent a real world cross sectionsome were operated recently, some were operated a long time ago. It is not clear when the 
blinding pathologies developed and whether something could have been done before, or at the time of surgery, to prevent them. The patient may have had good vision immediately following surgery, but does not now because of the subsequent onset of a blinding condition. In other cases it is possible that pre-existing posterior segment disease went undetected at the time of surgery, possibly because of a dense cataract; in effect, the patient was blind with cataract, not as a result of cataract. The surgical procedure itself is clearly implicated in some cases; if complications such as vitreous loss or wound leak had been avoided, the visual outcome would have been better.

VF and QOL outcomes parallel visual acuity outcomes. VF and QOL scores were significantly associated with visual acuity in both unoperated and operated cases (evidence as to the validity of the questionnaires). Although interviewers did not have access to visual acuity measurements, they were not masked as to the patient's general vision status. It is not known whether, or to what extent, this could have contributed to the favourable correlation between visual acuity and VF/QOL responses.

There was substantial variation in VF/QOL scores among respondents, even among those with similar vision status. (The interobserver reliability problems among the Lumbini interviewers may have contributed to this variation.) Vision and its impact on activities of daily living apparently goes far beyond what is measurable in the clinic with the Snellen chart. Variation in VF/QOL scores was only partially explained by differences in age, zone and, to a lesser extent, sex. A myriad of other factors pertinent to daily living undoubtedly come into play.

VF/QOL mean scores for the cataract operated group were not even at the levels in the unilateral blind group. For those cataract operated individuals achieving normal or near normal visual acuity in both eyes, $\mathrm{VF} / \mathrm{QOL}$ scores were close to, but not at, the levels of the nor- mal unoperated control group. A larger sample, adjusted for age, sex, and other socioeconomic variables, is required to investigate whether the cataract patient returns fully to normal VF/QOL status when vision is restored.

The study sample size was also too small to investigate differences in outcomes between aphakics and pseudophakics. A recently completed clinical trial involving 3400 patients in Madurai, India demonstrated that extremely favourable visual acuity and VF/QOL outcomes can be achieved with cataract surgery, with ECCE/IOL having an advantage over ICCE with spectacles. ${ }^{7}$ Considering what is apparently possible (albeit under the controlled environment of a clinical research study), it is clear that in the quest to reducing cataract blindness more attention must be given to the need for improving cataract surgery outcomes. This is important if patient satisfaction and gain in the expenditure of scarce resources are to be maximised.

The authors wish to thank Marriam Bright, National Eye Institute, for clerical assistance in preparing this manuscript. Thanks are also due to Dr R P Pokharel, Nepal Netra Jyoti Sangh, and staff at the Fatah-Bal and Rana-Ambika hospitals for facilitating the study.

Work supported in part by the World Health Organisation under National Institutes of Health contract N01-EY-2103.

1 Pokharel GP, Regmi G, Shrestha SK, et al. Prevalence of blindness and cataract surgery in Nepal. Br 7 Ophthalmol 1998;82:600-5

2 Thylefors B, Negrel AD, Pararajasegaram R, et al. Global data on blindness. Bull World Health Organ 1995;73:11521.

3 Limburg H, Kumar K, Bachani D. Monitoring and evaluatng cataract intervention in India. $\mathrm{Br} \mathscr{f}$ Ophthalmol 1996;80:951-5.

4 Ellwein LB, Kupfer C. Strategic issues in preventing cataract blindness in developing countries. Bull World cataract blindness in developin

5 Fletcher AE, Ellwein LB, Selvaraj S, et al. Measurements of vision function and quality of life in patients with cataracts in Southern India. Arch Ophthalmol 1997;115:767-74.

6 Kazis LE, Anderson JJ, Meenan RF. Effect sizes for interpreting changes in health status. Med Care 1989;27: S178-89.

7 Prajna NV, Chandrakanth KS, Kim R, et al. The Madurai intraocular lens study: II clinical outcomes. Am f Ophthal$\mathrm{mol}$ (in press). 